\title{
An Open-Label, Single-Dose, Crossover Study of the Pharmacokinetics and Metabolism of Two Oral Formulations of 1-Octanol in Patients with Essential Tremor
}

\author{
Fatta B. Nahab - Loretta Wittevrongel - Dominic Ippolito • Camilo Toro • \\ George J Grimes • Judith Starling • Gopal Potti • Dietrich Haubenberger • \\ Daniel Bowen • Peter Buchwald • Chuanhui Dong • Daniel Kalowitz • Mark Hallett
}

Published online: 19 May 2011

(C) The American Society for Experimental NeuroTherapeutics, Inc. (Outside the U.S.) 2011

\begin{abstract}
Existing therapeutic options for management of essential tremor are frequently limited by poor efficacy and adverse effects. Likely the most potent tremor suppressant used is ethanol, although its use is prohibitive due to a brief
\end{abstract}

Electronic supplementary material The online version of this article (doi:10.1007/s13311-011-0045-1) contains supplementary material, which is available to authorized users.

F. B. Nahab $(\bowtie) \cdot$ C. Dong

Department of Neurology,

University of Miami Miller School of Medicine,

Miami, FL 33136, USA

e-mail: fnahab@med.miami.edu

L. Wittevrongel - D. Ippolito - C. Toro - D. Haubenberger •

M. Hallett

Human Motor Control Section, Medical Neurology Branch, NINDS/NIH,

Bethesda, MD 20892, USA

G. J. Grimes $\cdot$ J. Starling $\cdot$ G. Potti

Pharmaceutical Development Section, NIH,

Bethesda, MD 20892, USA

D. Haubenberger

Department of Neurology, Medical University of Vienna,

Vienna, Austria

D. Bowen

Wake Forest University,

Winston-Salem, NC 27106, USA

P. Buchwald

Department of Molecular and Cellular Pharmacology,

University of Miami Miller School of Medicine,

Miami, FL 33136, USA

D. Kalowitz

Albert Einstein College of Medicine,

Bronx, NY 10461, USA therapeutic window, and the obvious implications of excessive alcohol use. Longer-chain alcohols have been shown to suppress tremor in harmaline animal models, and appear to be safe and well tolerated in 2 prior studies in humans. Here we report on the findings of a phase I/II study of 1-octanol designed to explore pharmacokinetics, efficacy, and safety. The most significant finding was the identification of octanoic acid as the product of rapid 1octanol metabolism. Furthermore, the temporal profile of efficacy closely matches the plasma concentration of octanoic acid. Therefore, these findings identify a novel class of compound (e.g., carboxylic acids) with tremor suppressive properties in ET. Administration of 1-octanol also appears to be safe based on various measures collected. Essential tremor (ET) is the most common tremor disorder, with tremors occurring during static posturing or movement. These tremors are known to briefly improve in many cases after alcohol (ethanol) consumption. Two previous studies of a longer chain alcohol, 1-octanol, have demonstrated longer duration tremor-suppressive effects without the occurrence of intoxication. The aim of this study was to characterize the pharmacokinetics of 1-octanol and its primary metabolite octanoic acid using two formulations, along with additional safety and efficacy measures. Participants with proven ethanol-responsive ET were recruited into 1 of 2 parts: (part A) a dose escalation study $(1-64 \mathrm{mg} / \mathrm{kg}$; $\mathrm{n}=4)$, and (part B) a fixed dose $(64 \mathrm{mg} / \mathrm{kg} ; \mathrm{n}=10)$ balanced, open-label crossover design. Two participants in part B then completed an exploratory part $\mathrm{C}$ evaluating $128 \mathrm{mg} / \mathrm{kg}$. Plasma samples were collected at 10 intervals during a 6-hour period postingestion. Efficacy was assessed using spirography, whereas safety was assessed with electrocardiograms, vital signs, adverse effects surveys, and an intoxication assessment. Plasma concentrations of 1-octanol were detect- 
able at low levels whereas octanoic acid (OA) concentrations were approximately 100-fold higher. The half-life of OA was 87.6minutes. This was matched by a clinical reduction in tremor severity of $32 \%$ at 90 minutes, assessed using spirography. The safety profile was favorable, with the most commonly reported adverse effect being dysgeusia (38\%). Early detection and higher plasma concentrations of OA are a product of rapid metabolism of 1-octanol.OA pharmacokinetics mirrored the timing of clinical improvement. These findings provide preliminary evidence for a new class of compound that may be effective in the treatment of ET.

Keywords 1-Octanol - Octanoic acid Essential tremor. Alcohol $\cdot$ Therapeutics $\cdot$ Spirography

\section{Introduction}

Essential tremor (ET) is a progressive neurological disorder causing postural and kinetic tremors of the hands, head, voice, and legs. The leading hypothesis for ET pathophysiology is an unmasking of spontaneous oscillations originating in neurons of the inferior olive [1]. A helpful diagnostic feature of ET is the observed reduction of tremor amplitude after ethanol intake with preservation of the tremor frequency. Of those with ET who consume alcoholic beverages and are aware of their tremor effects, 74\% report having ethanolresponsive tremors [2]. The mechanism by which ethanol provides therapeutic benefit is unknown, although animal data suggest tremor reduction is mediated by decreased synchronization of inferior olive oscillations [3]. Unfortunately, the tremor-suppression effects of ethanoloccur at a dose close to its effective dose (ED50) for intoxication, or the dose at which $50 \%$ of individuals experience this effect [4].

Similar to ethanol, 1-octanol has been found to block lowthreshold calcium channels in the inferior olive using a rat model of ET [3]. Based on this background, Bushara et al. [5] undertook an initial placebo-controlled single dose (1 mg/ $\mathrm{kg}$ ) trial of 1-octanol in 12 patients with ET. Their finding showed 1-octanol was well tolerated, with tremor benefits observed for 15 to 90 minutes postdose compared with placebo. A second open-label dose escalation study was designed to evaluate the tolerability of higher oral doses of 1octanol and found no evidence of intoxication or serious adverse effects up to $64 \mathrm{mg} / \mathrm{kg}$ [6]. Efficacy was found at all dosages, and a trend toward a dose response was noted. Both studies attempted to characterize pharmacokinetics, but were unable to detect 1-octanol. The inability to detect 1-octanol led us to speculate that rapid metabolism to a daughter compound may be occurring. Metabolism of octanol is mediated by various enzymes, with alcohol dehydrogenase $(\mathrm{ADH})$ playing a major role. This pathway involves the conversion of octanol to its corresponding aldehyde (octanal) before forming octanoic acid; however, under in vitro incubation of octanol with ADH, octanal disappears rapidly ( $<5 \mathrm{~min}$ ) leaving only octanoic acid [7]. This led us to hypothesize that octanoic acid was the most likely stable metabolite. Both prior studies also used a capsule formulation that could break open as participants attempted to swallow the pills, leading to various reports of an unpleasant taste (dysgeusia) associated with the 1-octanol. Concern of the feasibility of future blinded clinical trials led us to develop an alternate formulation for testing.

The objective of this trial was to characterize the pharmacokinetics of 1-octanol and its hypothesized primary metabolite, octanoic acid, using two different formulations. We also assessed efficacy, tolerability, and safety in this sample of patients with ethanol-responsive ET.

\section{Methods}

\section{Patients}

Since the mechanism of action of 1-octanol is likely similar to that of ethanol, we recruited ET patients who reported an improvement in tremor with alcohol. Additional inclusion criteria included: limb-predominant tremors, ability to safely stop and remain off all medications used to treat ET for at least 4 half-lives, willingness to abstain from ethanol and caffeine intake for at least 48 hours prior to and during the study participation, and ability to fast for periods of as much as 12 hours during the study.

Patients were excluded if they hadsignificant abnormalities other than tremor on neurological examination, past or present alcohol abuse or dependence, acute or chronic severe medical conditions (such as renal failure), hepatic failure or lung disease, taking primidone (due to its extended half-life), other acute or chronic medications potentially influencing hepatic metabolism or central nervous system function, inability to temporarily discontinue tremor medication for the duration of the study, significant abnormalities on baseline screening laboratory tests, and cognitive impairment preventing informed consent. We also excluded patients younger than 21 years of age, women who were pregnant or lactating, anyone who did not wish to take a potentially intoxicating drug, and patients from far East Asian or Native American descent (due to their likelihood for possessing variant alleles of the genes for alcohol metabolism that could result in slower metabolism and potentially increase their sensitivity to alcohols and their metabolites).

\section{Study Design}

Participants were recruited from the clinic, the local community, and through various internet-based resources. 
Eligible subjects were brought to the National Institutes of Health Clinical Center in Bethesda, Maryland. Written informed consent was obtained from all participants for this protocol that was approved by the institutional review board of the National Institute of Neurological Disorders and Stroke (Study Identifier: NCT00102596) and the Food and Drug Administration under investigational new drug status (IND\#61864 and \#68751).

The initial screening visit included a complete medical history and neurological examination, laboratory screening (complete blood count with differential, electrolytes, renal function, liver function, ethanol level, coagulation profile, urinalysis, urine pregnancy test), and electrocardiogram. The tremor examination was videotaped and severity assessed with the Fahn-Tolosa-Marin Tremor Rating Scale [8] and digital spiral analysis using a penbased Wacom tablet (Wacom Intuos 3 Model PTZ-930, Wacom Technology Corp., Vancouver, WA). Then patients judged to have ET based on consensus criteria [9] underwent an ethanol challenge to assess the degree of tremorresponsiveness. Subjects could receive up to three $50 \mathrm{ml}$ servings of ethanol ( $40 \%$ by volume) at 30-minute intervals. Tremor amplitude was assessed at 15-minute intervals by spirography time series analysis and portions of the tremor rating scale. Patients demonstrating ethanolresponsive tremors based on clinical assessment and reduction in tremor amplitudes on spirography were then eligible to participate in the inpatient study.

The inpatient study consisted of 2 parts (i.e., parts A and B). In part A, a dose escalation (1-64 $\mathrm{mg} / \mathrm{kg}$ ) study was used to validate the assay, and in part $\mathrm{B}$, a fixed dose (64 mg/kg) balanced, open-label crossover study was used to assess pharmacodynamics of the 2-drug formulations for the study. In both study parts, subjects fasted overnight for 6 hours before receiving 1-octanol at 6 AM. Four patients participated in part A, and 10 patients in part B. Sample size estimation was based on ethanol studies due to the lack of 1-octanol pharmacokinetic data. At the completion of these studies, and we added an exploratory part $\mathrm{C}$ using the same study design as part $\mathrm{B}$, but evaluating a $128 \mathrm{mg} / \mathrm{kg}$ dose in 2 patients who participated in part B.

We evaluated 2 formulations for this study. The first formulation, produced by the Pharmaceutical Development section of the National Institutes of Health, consisted of 1octanol adsorbed to microcrystalline cellulose, NF (Avicel PH 102, FMC Corp., Philadelphia, PA), and fine particle silica (Sipernat 50S, Evonik Degussa Corp., Parsippany, $\mathrm{NJ}$ ) and encapsulated in $50 \mathrm{mg}$ and $250 \mathrm{mg}$ dosages. This formulation (CEL) was used in the two prior studies of 1octanol $[5,6]$. A second formulation consisted of a soft-gel capsule containing 1-octanol embedded in soybean oil at $50 \mathrm{mg}$ and $800 \mathrm{mg}$ dosages (Best Formulations Inc, City of Industry, CA). This formulation (SOY) was developed due to concerns about the instability of the CEL formulation during swallowing and the potentially greater likelihood for participants to report dysgeusia if the capsule fractured prematurely.

Plasma samples were collected from a peripherally inserted central catheter at 10 time points during a 6-hour period after 1-octanol ingestion (Table 1). Because subjects received a weight-adjusted dose, the number of pills for each individual varied. Time of ingestion was defined as the time-point when the first pill was swallowed. After collection, blood samples were coded, centrifuged, and cooled on an hourly basis, then aliquoted and stored at $-80^{\circ} \mathrm{C}$ until shipped to the laboratory for processing. Samples were batch analyzed with high-pressure liquid chromatography with mass spectrometry using standard methods. Based on pre-clinical validation studies, the assay linear range of detection was 20 to $1000 \mathrm{ng} / \mathrm{ml}$ for both 1 octanol and octanoic acid.

Efficacy was assessed by tremor amplitude change via spirography time series analysis using a commercially available digitizing tablet, connected to a laptop. Subjects were asked to draw Archimedes spirals in between the lines of a 5-loop template spiral with each hand at multiple time points throughout the observation period. Tremor amplitudes were calculated at the tremor frequency peak after fast Fourier transformation of the spirography time series using published methodology [10, 11].

Safety measures included electrocardiograms, vital signs, and anadverse effects survey [12]. Potential signs of intoxication were assessed using an alcohol intoxication scale; six different domains were potentially affected by intoxication (sedation, speech, finger tapping, finger-tonose test, gait, tandem gait) and were examined, with each domain graded from 0 to $4(0=$ normal and $4=$ maximum intoxication). Assessments were made at baseline and at regular intervals after treatment (Table 1). At the completion of the inpatient testing, peripherally inserted central catheter lines were removed and participants were discharged after a final safety assessment. An outpatient telephone follow-up was conducted 1 week after discharge to assess for remote adverse effects.

\section{Statistical Analysis}

Pharmacokinetic analyses of the data for 1-octanol and octanoic acid, were performed using standard noncompartmental analyses with Phoenix WinNonlin 6.0 (Pharsight Corp., St. Louis, MO). Nominal times were used in all calculations; all actual times were close enough not to cause any significant changes in any of the pharmacokinetics (PK) estimates. Values for maximum plasma concentration, $C_{\max }$, and the time to reach maximum concentration, $t_{\max }$, were obtained directly from the concentration-time data. 


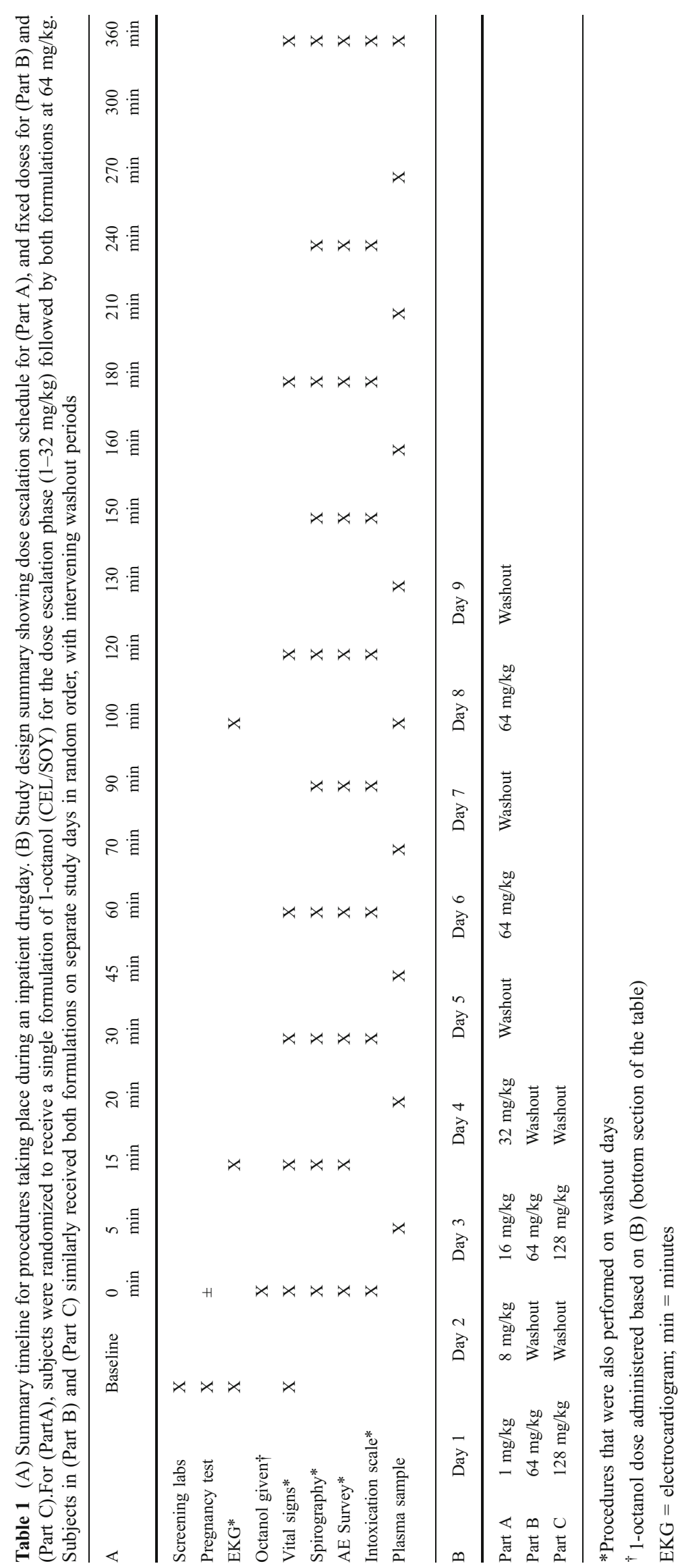


Terminal elimination rate constants $\left(\lambda_{\mathrm{z}}\right)$ were obtained using log-linear regression on the terminal phase, as selected by the default WinNonlin (Pharsight Corp.) procedure that uses the largest number of last points with nonzero concentrations that maximize the adjusted $r^{2}$ of the corresponding regression [13]. Elimination half-lives were then calculated using the standard $t_{1 / 2}=\ln (2) / \lambda_{\mathrm{z}}$ relationship. Areas under the curve (AUCs) were calculated using the linear trapezoidal method. The area under the curve to last concentration $\left(\mathrm{AUC}_{0-\mathrm{t}}\right)$ was calculated from time 0 to time of the last measurable concentration $\left(C \mathrm{p}_{\mathrm{L}}\right)$. The area under the curve to time infinity $\left(\mathrm{AUC}_{0-\infty}\right)$ was calculated as $\mathrm{AUC}_{0-\infty}=\mathrm{AUC}_{0-\mathrm{t}}-\mathrm{Cp}_{\mathrm{L}} / \lambda_{\mathrm{z}}$. The apparent volume of distribution $\left(V_{\mathrm{d}} / F\right)$ based on the terminal phase was calculated as $V_{\mathrm{d}} / F=\operatorname{dose} /\left(\lambda_{\mathrm{z}} \times \mathrm{AUC}_{0-\infty}\right)$, and the apparent clearance $\mathrm{CL} / F$ was be calculated as dose/AUC $\left.{ }_{0-\infty}\right)$. Because 1-octanol concentrations were undetectable or very low for the majority of patients, no $V_{\mathrm{d}}$ or CL calculations were done for 1-octanol and a set of formal $V_{\mathrm{d}}$ or CL estimates were made for the acid metabolite assuming full conversion of the parent compound. Data for each individual were fitted separately, and averages \pm standard deviations were calculated for each group. Concentration data below the lower limit of quantitation (20 $\mathrm{ng} / \mathrm{ml}$ for both compounds) were considered as zero.

For statistical analysis of efficacy, the spiral tremor amplitude of the right hand at each timepoint after drugadministration was normalized to each baseline, which was recorded on each study day before drug administration. A repeated measures 2 factor analysis of variance model was performed to assess for differences related to time (each time point) and formulation (CEL/SOY). As baseline was set to 1 without variance across subjects, only time points after administration were included in the model. Missing datapoints were imputed using the "last observation carried forward" method. The Kolmogorov-Smirnov test was applied to test for normal distribution. In case of significant effects, post-hoc comparisons were made using pairedsample $t$ tests. All comparisons were corrected for multiple testing (Bonferroni). A statistical level of $p<0.05$ was considered significant. Electrocardiogramparameters (heart rate, PR interval, and QTc) collected at multiple time points (baseline, 15 minutes, 100 minutes, and 24 hours) were compared using a mixed-effects model. Descriptive statistics were performed for all reported adverse effects.

Role of the Funding Source

The intramural program of the National Institute of Neurological Disorders and Stroke provided primary funding for this work. A collaborative research and development agreement between National Institute of Neurological Disorders and Stroke and Ariston Pharmaceuticals provided expertise and funding for assay development, sample testing, and support for a research coordinator.

\section{Results}

Twenty-one patients were screened and 14 patients completed the study (Fig. 1). Table 2 provides a summary of patient demographics. The study population consisted of varying disease severities, with the vast majority reporting a family history of ET, and all patients reported alcoholresponsive tremors because this was a criterion for enrollment. Tremor alcohol-responsiveness was clinically confirmed during the screening visit for all subjects enrolled.

The assays that were used detected both 1-octanol and octanoic acid successfully. Only very low 1-octanol concentrations were present in all participants, and clearly quantifiable levels $(>50 \mathrm{ng} / \mathrm{ml})$ were only present in a minority of participants in the 2 highest-dose groups (64 and $128 \mathrm{mg} / \mathrm{kg}$ ). Conversely, octanoic acid concentrations were detectable and quantifiable at all time points. In general, 1-octanol levels paralleled octanoic acid levels (Fig. 2), but because of the large number of 1-octanol data below the lower limit of quantitation at all dose levels, no quantitatively reliable analyses could be performed for 1octanol. Concentrations, $\mathrm{AUC}$, and $\mathrm{C}_{\max }$ values were approximately 100 -fold higher for the metabolite (octanoic acid) than the parent compound (1-octanol), suggesting that the effects of 1-octanol were mediated almost entirely by its metabolite. Furthermore, the metabolite appeared quickly in the plasma, with detectable octanoic acid concentrations seen within 5 minutes of oral ingestion in most individuals, and remained at quantifiable levels in all participants at the last sampling time (at 6 hours).

Because1-octanol concentrations were so low for the majority of participants, meaningful PK analysis could be performed only for octanoic acid (Table 3). The dose escalation part of the study $(1-32 \mathrm{mg} / \mathrm{kg})$ also contained

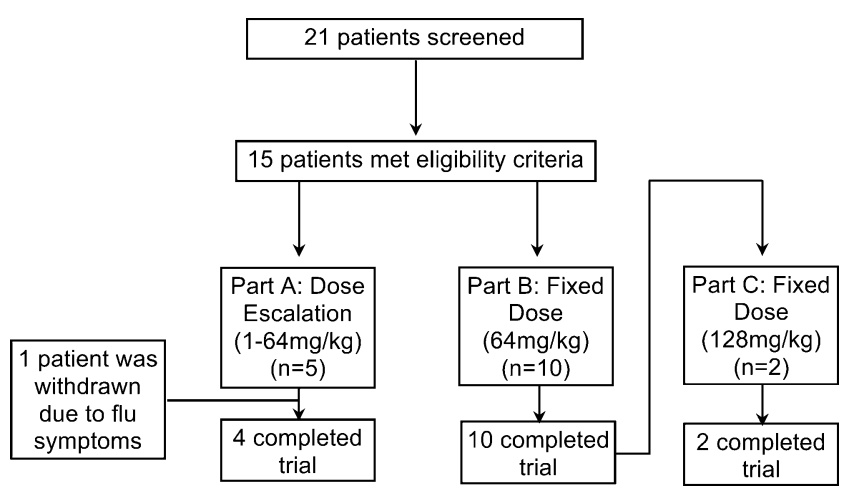

Fig. 1 Patient flow profile 
Table 2 Baseline demographic characteristics

\begin{tabular}{ll}
\hline Mean age (Standard deviation) & $68.1(8.1)$ \\
Gender, n (\%) & \\
Male & $9(60 \%)$ \\
Female & $6(40 \%)$ \\
Mean height, cm (Standard deviation) & $169.6(12.0)$ \\
Mean weight, kg (Standard deviation) & $82.9(20.1)$ \\
Mean body mass index (Standard deviation) & $28.6(4.7)$ \\
Mean age of onset, years (Standard deviation) & $39.5(18.6)$ \\
Mean disease duration, years (Standard deviation) & $28.7(17.2)$ \\
History of alcohol-responsive tremors, n (\%) & $15(100 \%)$ \\
Mean \# servings of alcohol (50 ml of 40\% ethanol) required for tremor response & $2.1(0.3)$ \\
Family history of Essential tremor, n (\%) & $13(87 \%)$ \\
Mean tremor rating scale score (Standard deviation) & 1 \\
\end{tabular}

\footnotetext{
${ }^{1}$ Data for patients $1-5$ not available

$\mathrm{n}=$ number of patients
}

meaningfully evaluated, elimination showed no dosedependence, also confirming an essentially linear PK (e.g., the elimination constant $\lambda_{z}$ has a value of around $\left.0.01 \mathrm{~min}^{-1}\right)$. The average elimination half-life $\left(\mathrm{t}_{1 / 2}\right)$ of all doses and formulations is 87.6 minutes for octanoic acid.

Assessment of the clinical response using spirography time course analysis compared every study timepoint for participants to their own baseline tremor severity of the right hand (Fig. 4). Normalized spirography tremor scores were normally distributed. The repeated measures of analysis of variance showed significant changes from baseline across time (F3.014; $p=0.006)$. Post-hoc comparisons showed a significant reduction in tremor scores at 90 ( $p=0.002), 120(p=0.006), 150(p=0.03)$, and 180 minutes $(p=0.003)$. We found no significant differences based on formulation type $(p=0.8)$ and no significant interaction between time and formulation $(p=0.3)$. We also found no rebound tremor up to 24-hours postdose.

Assessment of potential cardiovascular effects of 1-octanol using baseline and postdose electrocardiograms found
Fig. 2 Concentration time profiles for 1-octanol (dashed lines) and octanoic (oct.) acid (solid lines) for both formulations (CEL: green; SOY: blue) after a $64-\mathrm{mg} / \mathrm{kg}$ dose. Data are mean \pm standard error of the mean for all subjects $(\mathrm{n}=12$; balanced crossover design); note that compared to octanoic acid, octanol concentrations are shown on a magnified scale (right vertical axis)

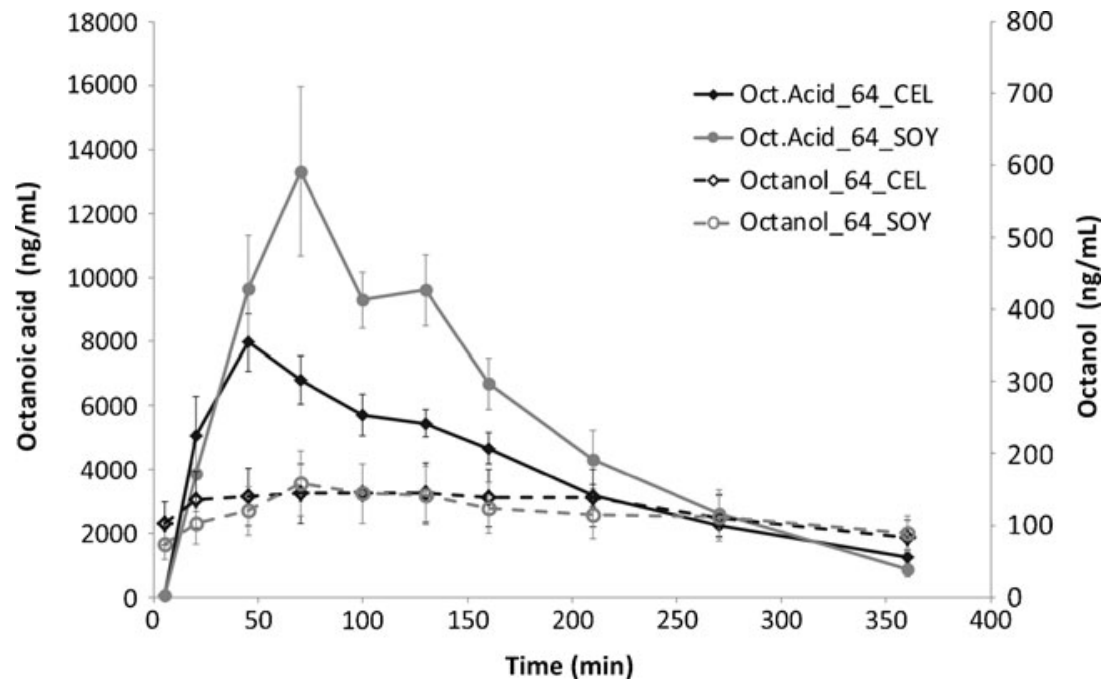


Table 3 Summary of PK parameters for octanoic acid for all doses and formulations

\begin{tabular}{lllllllll}
\hline Form. & Dose $(\mathrm{mg} / \mathrm{kg})$ & $N$ & $C_{\max }(\mathrm{ng} / \mathrm{mL})$ & $T_{\max }(\min )$ & $\mathrm{AUC}_{6 \mathrm{~h}}(\mathrm{ng} \min / \mathrm{mL})$ & $\lambda_{\mathrm{z}}(1 / \mathrm{min})$ & $T_{1 / 2}(\mathrm{~min})$ & $\mathrm{AUC} C_{\infty}(\mathrm{ng} \mathrm{min} / \mathrm{mL})$ \\
\hline CEL & 1 & 2 & 385.7 & 20.0 & 46231.0 & 0.00154 & 450.3 & 94938.2 \\
CEL & 4 & 1 & 925.0 & 70.0 & 133859.2 & 0.01070 & 64.8 & 140889.1 \\
CEL & 8 & 2 & 2820.0 & 20.0 & 213535.5 & 0.00901 & 85.6 & 222307.1 \\
CEL & 16 & 2 & 3829.8 & 12.5 & 312371.2 & 0.00825 & 89.1 & 323631.3 \\
CEL & 32 & 3 & 4809.2 & 45.0 & 761165.0 & 0.01316 & 61.1 & 792446.1 \\
CEL & 64 & 12 & 9143.4 & 72.9 & 1398164.9 & 0.00733 & 102.7 & 1620687.7 \\
CEL & 128 & 2 & 25019.2 & 32.5 & 4810868.2 & 0.00500 & 148.3 & 5564892.8 \\
SOY & 1 & 3 & 607.3 & 36.7 & 39714.5 & 0.00150 & 558.4 & 87366.7 \\
SOY & 4 & 1 & 1999.3 & 45.0 & 112874.8 & 0.00566 & 122.4 & 117814.1 \\
SOY & 8 & 3 & 3434.7 & 53.3 & 247018.6 & 0.00874 & 86.3 & 256278.7 \\
SOY & 16 & 2 & 8360.7 & 60.0 & 543195.6 & 0.01168 & 67.9 & 550814.6 \\
SOY & 32 & 3 & 9737.0 & 93.3 & 954747.0 & 0.01111 & 69.2 & 980749.0 \\
SOY & 64 & 12 & 15548.2 & 81.7 & 1992316.9 & 0.01221 & 62.0 & 2078459.6 \\
SOY & 128 & 2 & 30070.8 & 100.0 & 4616032.5 & 0.01160 & 180.6 & 5581177.2 \\
\hline
\end{tabular}

$\lambda_{\mathbf{z}}=$ Terminal elimination rate constant; $\mathbf{A} \mathbf{U C}_{\infty}=$ area under the curve to time infinity; $\mathbf{A} \mathbf{U C}_{\mathbf{6 h}}=$ area under the curve at 6-hours; $\boldsymbol{C}_{\mathbf{m a x}}=$ Maximum plasma concentration; $\mathrm{CEL}=$ Cellulose formulation; Form. $=$ formulation; $\min =$ minutes; $N=$ number of subjects; PK $=$ Pharmacokinetics; SOY $=$ Soy formulation; $\boldsymbol{T}_{\mathbf{m a x}}=$ Time to maximum concentration; $\boldsymbol{T}_{\mathbf{1} / \mathbf{2}}=$ Elimination half-life

transient reductions in the heart rate at the 15-minute $(-4.2 \mathrm{bpm} ; p=0.02)$ and 100 -minute $(-3.7 \mathrm{bpm} ; p=0.04)$ timepoints (Table 4). No clinically significant bradycardiaor changes in blood pressure, respiratory rate, or temperature were observed. The adverse effects profile was similar to prior reports with dysgeusia being the most common, reported by $38 \%$ of participants with varying descriptions of bitter, acidic, sweet, or metallic flavors. Reports of dysgeusia were more common with the CEL formulation compared with SOY (62.5\% vs $37.5 \%$, respectively). Other adverse effects are summarized in Table 5. No significant signs of intoxication were observed on clinical evaluation,

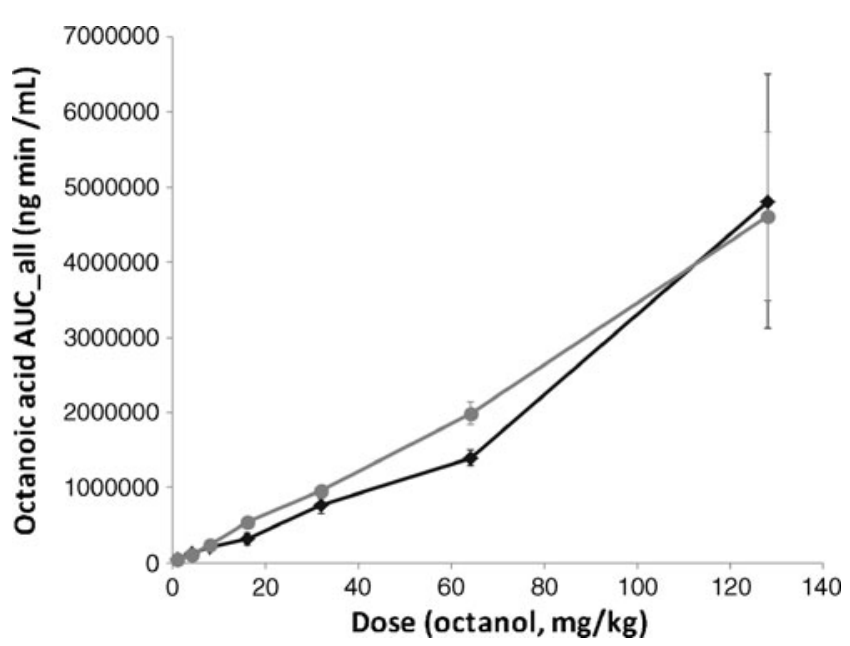

Fig. 3 Total octanoic acid area under the concentration $\left(\mathrm{AUC}_{6 \mathrm{~h}}\right)$ as a function of 1-octanol dose. Data are mean \pm standard error of the mean for all subjects at all dose levels in each formulation; the number of subjects at each dose level are as those summarized in Table 3 with only a single participant at a single time point $(64 \mathrm{mg} / \mathrm{kg}$, 240 minutes) during the entire study having transient, mild gait instability. No significant adverse effects were reported during the telephone survey 1 week after discharge. No serious adverse effects were drugrelated. Two unrelated serious adverse events included 1 patient who was withdrawn from the study after developing flu symptoms after study day 1 and having spontaneous resolution with a typical course ensuing for 2 days, while a second patient accidentally slipped and sustained a fractured rib during the 1-week follow-up period after completion of inpatient participation.

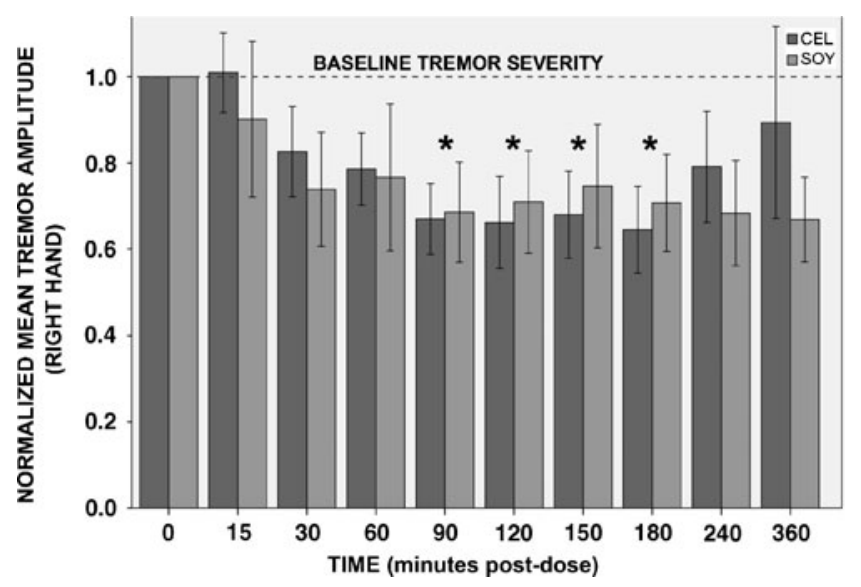

Fig. 4 Spirography mean tremor amplitudes for right hand vs time postoctanol dose $(64 \mathrm{mg} / \mathrm{kg})$. Time course of tremor improvement shows a maximal reduction by 90 to 180 minutes, independent of the formulation (CEL/SOY) and persistent effects beyond the study time window for the SOY formulation. Error bars represent standard error. *Statistical significance $(p<0.05)$ for each time-point vs baseline 


\section{Discussion}

Historic references to ET as a "benign tremor" have been supplanted by recent work showing nearly three quarters of affected individuals experience significant disability, decreased productivity and reduced quality of life [14]. Despite the diversity of nonsurgical pharmacologic options, adverse effects are common and 25 to $55 \%$ of patients gain no benefit from medical therapy [14]. Arguably the most effective treatment, ethanol is known to have potent anti-tremor properties [4], although routine use is prohibitive due to the high risk of intoxication and the serious medical, social, and legal consequences of chronic alcohol use.

1-octanol has been proposed as a potentially safer alternative alcohol due to its generally recognized as safe status by the Food and Drug Administration [15] and the lower effective dose required to reduce harmaline-induced tremor by $50 \%$ (ED50) of $0.26 \mathrm{mg} / \mathrm{kg}$ compared with $100 \mathrm{mg} / \mathrm{kg}$ of ethanol in animal models [3, 16]. Although animal data of 1-octanol and ethanol show similar acute toxicology profiles, 1 -octanol is approximately 5 to 6 times less potent in inducing intoxication [17-19]. These data would suggest an approximate 1-octanol ED50 for intoxication of $5.8 \mathrm{~g}$ for a single dose, close to our $64 \mathrm{mg} / \mathrm{kg}$ dosing, although we found no signs of intoxication at this level or in the 2 subjects we studied at $128 \mathrm{mg} / \mathrm{kg}$.

The most significant outcome of this work is the confirmation that 1-octanol is rapidly absorbed and metab-

Table 4 Electrocardiogram summary $(n=15)$

\begin{tabular}{lll}
\hline Parameter & Least square mean* $(\mathrm{SE})$ & Significance $(p)$ \\
\hline Heart rate & & \\
Baseline & $70.8(2.9)$ & Reference \\
15 min & $66.6(2.7)$ & 0.02 \\
100 min & $67.1(2.7)$ & 0.04 \\
$24 \mathrm{~h}$ & $72.4(2.7)$ & 0.38 \\
PR Interval (ms) & & \\
Baseline & $168.8(6.6)$ & Reference \\
15 min & $172.8(6.2)$ & 0.31 \\
100 min & $171.6(6.2)$ & 0.48 \\
$24 \mathrm{~h}$ & $168.6(6.2)$ & 0.97 \\
QTc (ms) & & \\
Baseline & $433.8(8.4)$ & Reference \\
15 min & $436.5(7.4)$ & 0.67 \\
100 min & $433.0(7.4)$ & 0.89 \\
24 h & $437.4(7.5)$ & 0.58 \\
\hline
\end{tabular}

*Adjusted for age, sex, period and formulation.

$\mathrm{h}=$ hours, $\min =$ minutes; $\mathrm{ms}=$ milliseconds; $\mathrm{n}=$ number of subjects; $\mathrm{SE}=$ Standard error
Table 5 Adverse events summary, n (\%)

Body system/organ class

CNS and PNS disorders

\begin{tabular}{ll} 
Headache & $5(24 \%)$ \\
Sedation & $2(10 \%)$ \\
Dizziness & $2(10 \%)$ \\
Worsening tremor & $2(10 \%)$ \\
Numbness & $1(5 \%)$ \\
Vertigo & $1(5 \%)$ \\
Gastrointestinal & \\
Dysgeusia & $8(38 \%)$ \\
Heartburn & $5(24 \%)$ \\
Gas/bloating & $5(24 \%)$ \\
Nausea & $4(19 \%)$ \\
Dry mouth & $4(19 \%)$ \\
Constipation & $3(14 \%)$ \\
Diarrhea & $2(10 \%)$ \\
Cardiovascular & \\
Hypotension & $1(5 \%)$ \\
Lightheadedness & $1(5 \%)$ \\
Respiratory & \\
Cough & $2(10 \%)$ \\
Psychiatric disorders & $1(5 \%)$ \\
Anxiety & \\
Insomnia & \\
Visual disorders & $1(5 \%)$ \\
Blurred vision & \\
Dry eyes & \\
Skin disorders & \\
Sweating & \\
\hline & \\
\hline
\end{tabular}

olized to octanoic acid. Octanol is a typical substrate for $\mathrm{ADH} 3$, which is highly expressed in gastric mucosa and helps to explain the rapid appearance of octanoic acid observed here [20]. Based on the pharmacokinetic data and spirography, the anti-tremor effects appear to be almost entirely mediated by octanoic acid, although the open-label design of our study and the administration of 1-octanol limits us from making this definitive conclusion. This is significant for a number of reasons. First, octanoic acid has a bioavailability of nearly $100 \%$ [21]. Second, reaching the plasma concentrations of octanoic acid achieved in this study would require a lower oral dose of octanoic acid than that of 1-octanol. Finally, octanoic acid also has Food and Drug Administration generally recognized as safe status and human dietary consumption of up to $710 \mathrm{mg} / \mathrm{kg}$ is considered safe [22].

The improvements in tremors we identified at the $64 \mathrm{mg} / \mathrm{kg}$ dosages were significant, with a mean improvement in tremor severity of $32.2 \%$ at 90 minutes and a sustained 
benefit $(33.2 \%)$ at 6 hours (SOY formulation). Furthermore, these improvements were clinically evident and confirmed by patient observations. The findings also underscore the importance of using an unbiased objective measure of tremor that is highly sensitive and does not rely on highlytrained raters to ensure validity [10].

The safety profile of 1 -octanol in this study was consistent with prior reports $[5,6]$ and showed 1-octanol to be well tolerated, with mild side effects, such as headache, gastrointestinal discomfort, and nausea in less than a quarter of participants. These symptoms, along with dysgeusia, are likely to be formulation-related and may be minimized by future formulations. Methodological shortcomings of this study include the small sample size, openlabel design, and indirect evidence for the therapeutic effects of octanoic acid. Future studies should build on this initial work to evaluate the efficacy and safety of direct administration of octanoic acid, as well as the long-term safety and efficacy of multiple daily dose administrations for the management of ET.

Acknowledgements The authors wish to acknowledge the International Essential Tremor Foundation and Tremor Action Network for assistance with patient recruitment, the 7SW Nursing Staff of the National Institutes of Health(NIH) Clinical Research Center, Elaine Considine and Barbara Kimber for their assistance in patient coordination, and David Luckenbaugh, MA (National Institute of Mental Health, NIH, Bethesda, MD) for statistical consultation.

This work was supported by the Intramural division of the National Institute of Neurological Disorders and Stroke and a collaborative research and development agreement with Ariston Pharmaceuticals; Dr. Haubenberger received research support through the National Institute of Neurological Disorders and Stroke (NINDS) Intramural Research Program and the Austrian Science Fund FWF (Erwin Schroedinger Fellowship, project no. J2783-B09); Dr. Hallett serves as Chair of the Medical Advisory Board and receives funding for travel from the Neurotoxin Institute; serves as Chair of the Medical Advisory Board of the Benign Essential Blepharospasm Foundation and Chair of the Medical Advisory Board of the International Essential Tremor Foundation; Dr. Hallett has received honoraria and/ or funding for travel for lectures or educational activities not funded by industry; and serves on Editorial Advisory Boards for Clinical Neurophysiology, Brain, Acta Neurologica Scandinavica, Journal of Clinical Neurophysiology, Italian Journal of Neurological Sciences, Medical Problems of Performing Artists, Annals of Neurology, Neurology and Clinical Neurophysiology, The Cerebellum, NeuroRx, Current Trends in Neurology, Faculty of 1000 Biology, Faculty of 1000 Medicine, Brain Stimulation, Journal of Movement Disorders (Korea), and World Neurology. Dr. Hallett may accrue revenue on U. S. Patent \#6,780,413 B2 (issued: August 24, 2004): immunotoxin (MAB-Ricin) for the treatment of focal movement disorders; U.S. Patent \#7,407,478 (issued: August 5, 2008): coil for magnetic stimulation and methods for using the same; receives royalties from publishing from Blackwell Publisher, Cambridge University Press, Springer Verlag, Taylor \& Francis Group, Oxford University Press, John Wiley \& Sons, and Elsevier; Dr. Hallett receives research support from Ariston Pharmaceuticals, National Institutes of Health (NIH)/NINDS (Intramural Program) and the U.S. Department of Defense (Army); Dr. Hallett has received license fee payments from the NIH (from Brainsway) for licensing the patent for the $\mathrm{H}$ - coil. Dr. Hallett's research at the NIH is largely supported by the NIH Intramural Program. Supplemental research funds come from the U.S. Army via the Henry Jackson Foundation, Ariston Pharmaceutical Company via a Cooperative Research and Development Agreement (CRADA) with the NIH, and the Kinetics Foundation via a Clinical Trials Agreement (CTA) with the NIH.Dr. Hallett is an inventor for patent applications of 1-octanol and octanoic acid held by NINDS/NIH.

Full conflict of interest disclosure is available in the electronic supplementary material for this article.

Disclosures Dr. Nahab has received research support from the International Essential Tremor Foundation, honoraria from GE Healthcare and Allergan, and is an inventor for patent applications of 1-octanol and octanoic acid held by NINDS/NIH.

\section{References}

1. Deuschl G, Raethjen J, Lindemann M, Krack P. The pathophysiology of tremor. Muscle Nerve 2001;24:716-735.

2. Koller WC, Busenbark K, Miner K. The relationship of essential tremor to other movement disorders: report on 678 patients. Essential Tremor Study Group. Ann Neurol 1994;35:717-723.

3. Sinton CM, Krosser BI, Walton KD, Llinas RR. The effectiveness of different isomers of octanol as blockers of harmaline-induced tremor. Pflugers Arch 1989;414:31-36.

4. Koller WC, Biary N. Effect of alcohol on tremors: comparison with propranolol. Neurology 1984;34:221-222.

5. Bushara K, Goldstein S, Grimes G, Burstein A, Hallett M. Pilot trial of 1-octanol in essential tremor. Neurology 2004;62:122-124.

6. Shill HA, Bushara KO, Mari Z, Reich M, Hallett M. Open-label dose-escalation study of oral 1-octanol in patients with essential tremor. Neurology 2004;62:2320-2322.

7. Hinson JA, Neal RA.An examination of octanol and octanal metabolism to octanoic acid by horse liver alcohol dehydrogenase. Biochimica et Biophysica Acta 1975;384:1-11.

8. Fahn S, Tolosa E, Marin C. Clinical rating scale for tremor. In: Jankovic J. Tolosa E, eds. Parkinson's disease and movement disorders, 2nd ed. Baltimore: Williams and Wilkins, 1993:271-280.

9. Bain P, Brin M, Deuschl G, et al. Criteria for the diagnosis of essential tremor. Neurology 2000;54(11 suppl 4):S7.

10. Haubenberger D, Kalowitz D, Nahab FB, et al. Validation of computerized essential tremor spiral analysis as outcome parameter for clinical trials in essential tremor. Mov Disord (under review).

11. Haubenberger D, Kalowitz D, Nahab FB, et al. Validity and reliability of computerized tremor spirography as outcome measure for clinical trials in essential tremor. Neurology 2010 (2):A349,P04.126

12. Cancer Therapy Evaluation Program. Common Terminology Criteria for Adverse Events, version 3.0; 2003. Available from: http://ctep.cancer.gov.

13. Gabrielsson J, Weiner D. Pharmacokinetic/pharmacodynamic data analysis: concepts and applications, 3rd ed. Apotekarsocieteten, ed. Stockholm: Swedish Pharmaceutical Press; 2000.

14. Louis E. Clinical practice. Essential tremor. NEJM 2001;345:887891.

15. Food and Drug Administration, 2000. Doc. No. \#1145. In: Food Safety Additive Profile and $\S 184.1025$.

16. Rappaport MS, Gentry RT, Schneider DR, Dole VP. Ethanol effects on harmaline-induced tremor and increase of cerebellar cyclic GMP. Life Sci 1984;34:49-56.

17. McCreery MJ, Hunt WA. Physico-chemical correlates of alcohol intoxication. Neuropharmacology 1978;17:451-461. 
18. Lyon RC, McComb JA, Schreurs J, Goldstein DB. A relationship between alcohol intoxication and the disordering of brain membranes by a series of short-chain alcohols. J Pharmacol Exp Ther 1981;218:669-675.

19. Wakabayashi T, Adachi K, Popinigis J. Effects of alkyl alcohols and related chemicals on rat liver structure and function. I. Induction of two distinct types of megamitochondria. Acta Pathol Jpn 1991;41:405-413.
20. Jelski W, Chrostek L, Szmitkowski M, Laszewicz W. Activity of class I, II, III, and IV alcohol dehydrogenase isoenzymes in human gastric mucosa. Dig Dis Sci 2002;47:1554-1557.

21. Schwabe AD, Bennett LR, Bowman LP. Octanoic acid absorption and oxidation in humans. J Appl Physiol 1964;19:335-337.

22. Traul KA, Driedger A, Ingle DL, Nakhasi D. Review of the toxicologic properties of medium-chain triglycerides. Food Chem Toxicol 2000;38:79-98. 